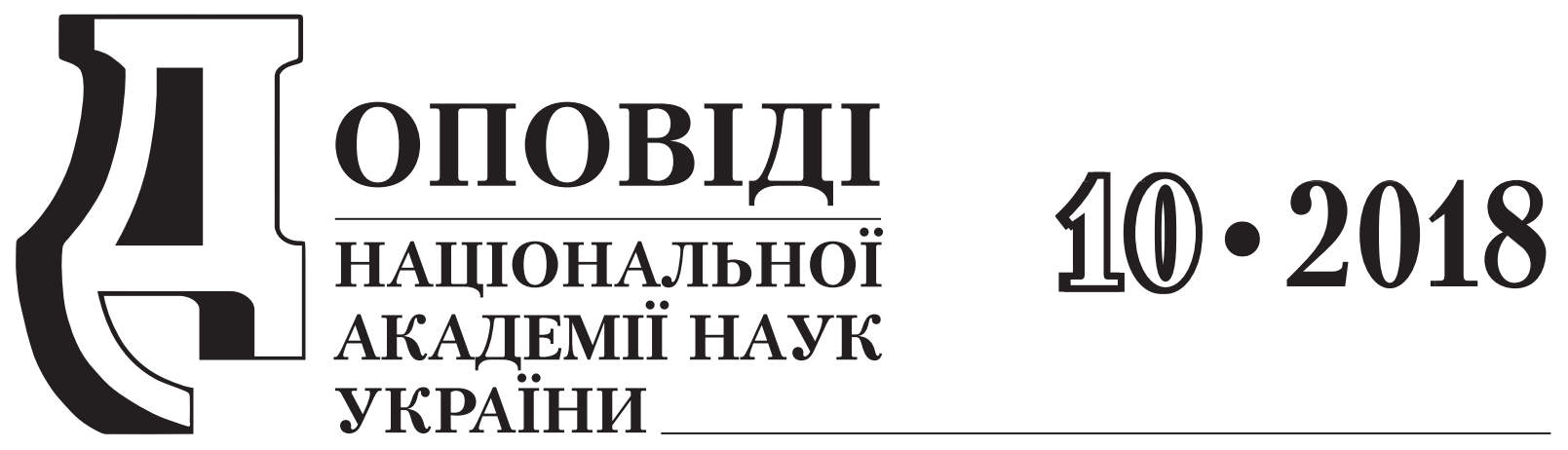

НАУКОВО-ТЕОРЕТИЧНИЙ ЖУРНАЛ • ЗАСНОВАНИЙ У 1939 р. • ВИХОДИТЬ ЩОМІСЯЦЯ • КИЇВ

\title{
REPORTS OF THE NATIONAL ACADEMY OF SCIENCES OF UKRAINE
}

\section{Зміст}

\section{МАТЕМАТИКА}

Михайлець B.А., Молибога В.Н. О лакунах в спектре оператора Хилла-Шредингера с сингулярным потенциалом.................

Gutlyanskǐ̌ V.Ya., Nesmelova O.V., Ryazanov V.I. On the regularity of solutions of quasilinear Poisson equations $\ldots \ldots \ldots \ldots \ldots \ldots \ldots \ldots$

\section{ІНФОРМАТИКА}

Панкратова Н.Д., Савченко I.О., Гайко Г.І., Кравець В.Г. Системний підхід до освоєння підземного простору мегаполісів в умовах невизначеностей та багатофакторних ризиків . . . . . Ustimenko V.A. On new symbolic key exchange protocols and cryptosystems based on a hidden tame homomorphism ...............

\section{MEXАНIКА}

Жбадинський І.Я. Взаємодія одноперіодичних податливих дискових еліптичної форми включень при падінні пружної гармонічної хвилі . . .

Каминский А.А., Курчаков Е.Е. Об эволюции зоны предразрушения у вершины трещины в нелинейном анизотропном теле . . . . . . . .

Трощенко В.Т., Хамаза Л.А. Стадии усталостного разрушения металлов и сплавов . . . . . . .

\section{Contents}

\section{MATHEMATICS}

Mikhailets V.A., Molyboga V.M. On spectral gaps of the Hill-Schrödinger operator with singular

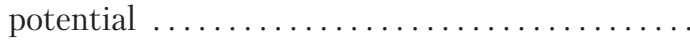

Gutlyanskǐ̌ V.Ya., Nesmelova O.V., Ryazanov V.I. On the regularity of solutions of quasilinear Poisson equations $\ldots \ldots \ldots \ldots \ldots \ldots \ldots \ldots \ldots$

\section{INFORMATICS}

Pankratova N.D., Savchenko I.O., Haiko H.I., Kravets V.H. A system approach to developing the underground space of metropolises under conditions of uncertainty and multifactor risks. . Ustimenko V.A. On new symbolic key exchange protocols and cryptosystems based on a hidden tame homomorphism ...............

\section{MECHANICS}

Zhbadynskyi I.Ya. Interaction of one-periodic compliant disk elliptic-shape inclusions under the action of an incident elastic time-harmonic

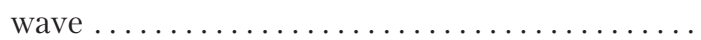

Kaminsky A.A., Kurchakov E.E. On the evolution of the prefracture zone near the crack tip in a nonlinear anisotropic body .............. Troshchenko V.T., Khamaza L.A. Stages of fatigue 56

(c) Національна академія наук України, 2018 


\section{НАУКИ ПРО ЗЕМЛЮ}

Орлюк М.I., Роменещь А.О. Просторово-часова збуреність геомагнітного поля ряду територій північної та південної півкуль Землі ........

\section{XIMI9}

Крупская Т.В., Ругаль А.А., Туров В.В. Особенности связывания воды в композитных системах $\mathrm{SiO}_{2}$ /левомицетин и $\mathrm{SiO}_{2}$ /левомицетин/

AM1

Москвіна В.С., Красилов І.В., Хиля В.П. Синтез оксимів піранонеофлавонів та спіропіранонеофлавонів. ........................

\section{БІологія}

Бузіашвілі А.Ю., Ємещь А.І. Отримання ліній рослин картоплі та томатів з геном лактоферину людини $\ldots \ldots \ldots \ldots \ldots \ldots \ldots \ldots$

Колесников Я.С., Кретинін С.В. Роль специфічних ізоферментів фосфоліпази D у реалізації біологічного ефекту жасмонової кислоти в реакціях рослин на дію стресів . . . . . . . . . . . .

Співак М.Я., Рибалко С.Л., Старосила Д.Б., Завелевич М.П., Олексієнко І.П., Дядюн С.Т., Руденко А.В., Атаманюк В.П. Оцінка впливу флавоноїдвмісного препарату Протефлазід на моделі папіломавірусної інфекції in vitro . . . .

\section{МЕДИЦИНА}

Дёмина Э.А. Оценка влияния профессионального облучения на цитогенетические показатели лимфоцитов периферической крови . . .

\section{ЕКОлогІя}

Бабенко Л.М., Водка М.В., Акимов Ю.Н., Бабенко А.В., Косаковская И.В. Влияние экстремальных температур на ультраструктуру митохондрий клеток мезофилла листьев Triticum spelta...........................

\section{GEOSCIENCES}

Orlyuk M.I., Romenets A.O. Spatial-temporal perturbation of the geomagnetic field of certain territories in the northern and southern hemi-

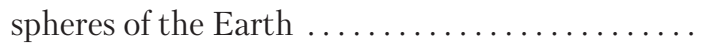

\section{CHEMISTRY}

Krupskaya T.V., Rugal A.O., Turov V.V. Water bounding peculiarities in $\mathrm{SiO}_{2}$ /laevomycetin and $\mathrm{SiO}_{2}$ /laevomycetin/AM1 composite systems ...

Moskvina V.S., Krasylov I.V., Khilya V.P. Synthesis of oximes of pyranoneoflavons and spiropy-

ranoneoflavons

\section{BIOLOGY}

Buziashrili A.Yu., Yemets A.I. The obtaining of tomato and potato plants with human lactoferrin

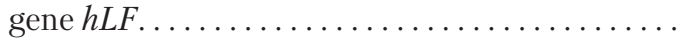

Kolesnikov Ya.S., Kretynin S.V. Role of specific phospholipase D isoenzymes in biological action of jasmonic acid during plant stress responses ...

Spivak M.Ya., Rybalko S.L., Starosyla D.B., Zavelevich M.P., Oleksiienko I.P., Diadiun S.T., Rudenko A.V., Atamaniuk V.P. Study of the effects of flavonoid-containing composition Proteflazid on modeled papillomavirus infection in vitro ......

\section{MEDICINE}

Domina E.A. Evaluation of the effect of professional irradiation on cytogenetic parameters of peripheral blood lymphocytes ...............

\section{ECOLOGY}

Babenko L.M., Vodka M.V., Akimov Yu.N., Babenko A.V., Kosakivska I.V. Extreme temperature effects on the ultrastructure of mitochondria of mesophyll cells in Triticum spelta leaves......... 
doi: https://doi.org/10.15407/dopovidi2018.10.003

УДК 517.984.5

\section{В.А. Михайлец, В.Н. Молибога}

Институт математики НАН Украины, Киев

E-mail: mikhailets@imath.kiev.ua,molyboga@imath.kiev.ua

\section{О лакунах в спектре оператора Хилла-Шредингера с сингулярным потенциалом}

Представлено членом-корреспондентом НАН Украины А.Н. Кочубеем

Исследуется непрерывный спектр оператора Хилла-Шредингера в гильбертовом пространстве $L^{2}(\mathbb{R})$. Предполагается, что потенциал оператора принадлежит классу Соболева $H_{\mathrm{loc}}^{-1}(\mathbb{R})$. Найдень условия, при которых последовательность длин спектральных лакун: а) ограничена; б) стремится к нулю. Особо изучен случай, когда потенциал является вещественной мерой Радона на $\mathbb{R}$.

Ключевые слова: оператор Хилла, непрерывный спектр, спектральная лакуна, сильно сингулярный потенииал.

1. Рассмотрим дифференциальное выражение Хилла-Шредингера

$$
S(q) u=-u^{\prime \prime}+q(x) u, \quad x \in \mathbb{R}
$$

с вещественной 1-периодической обобщенной функцией $q(\cdot)$ из негативного пространства Соболева $H_{\text {loc }}^{-1}(\mathbb{R})$. Ее ряд Фурье-Шварца имеет вид

$$
q(x)=\sum_{k \in \mathbb{Z}} \hat{q}(k) e^{i k 2 \pi x},
$$

где коэффициенты ряда удовлетворяют условиям

$$
\sum_{k \in \mathbb{Z}}(1+2|k|)^{-2}|\hat{q}(k)|^{2}<\infty
$$

и

$$
\hat{q}(k)=\overline{\hat{q}(-k)}, \quad k \in \mathbb{Z}
$$

Дифференциальное выражение (1) корректно определяется как квазидифференциальное [1-5]. Оно задает в сепарабельном комплексном гильбертовом пространстве $L^{2}(\mathbb{R})$ оператор Хилла-Шредингера $S(q)$, который определен на плотном в $L^{2}(\mathbb{R})$ множестве функций

$$
\operatorname{Dom}(S(q))=\left\{u \in H^{1}(\mathbb{R}) \mid-u^{\prime \prime}+q(x) u \in L^{2}(\mathbb{R})\right\}
$$

(C) В.А. Михайлец, В.Н. Молибога, 2018 
и действует по формуле (1). При этом выражения $u^{\prime \prime}$ и $q(x) u$ понимаются в смысле распределений.

Оператор $S(q)$ самосопряжен в гильбертовом пространстве $L^{2}(\mathbb{R})$ и полуограничен снизу (см., например, [4], где также приведены иные эквивалентные определения этого оператора). Спектр оператора $S(q)$ абсолютно непрерывен и имеет зонную структуру: его спектральные зоны перемежаются со спектральными лакунами [2-5]. При этом концы спектральных лакун $\left\{\lambda_{0}^{+}(q), \lambda_{n}^{ \pm}(q)\right\}_{n=1}^{\infty}$, как и в классическом случае суммируемого с квадратом потенциала, удовлетворяют неравенствам [4, теорема C]:

$$
-\infty<\lambda_{0}^{+}(q)<\lambda_{1}^{-}(q) \leqslant \lambda_{1}^{+}(q)<\lambda_{2}^{-}(q) \leqslant \lambda_{2}^{+}(q)<\lambda_{3}^{-}(q) \leqslant \lambda_{3}^{+}(q) \cdots,
$$

где $\left\{\lambda_{0}^{+}(q), \lambda_{n}^{ \pm}(q)\right\}_{n=1}^{\infty}$ при четных/нечетных $n$ являются собственными значениями соответствующих периодической/полупериодической граничных задач на единичном отрезке [6, 7].

2. Обозначим через

$$
\gamma_{q}(n):=\lambda_{n}^{+}(q)-\lambda_{n}^{-}(q) \geqslant 0, \quad n \in \mathbb{N}
$$

длины спектральных лакун оператора $S(q)$. Некоторые из лакун могут вырождаться. Если $q(\cdot) \in L_{\mathrm{loc}}^{2}(\mathbb{R})$, то, как известно, длины спектральных лакун стремятся к нулю, а скорость сходимости возрастает вместе с гладкостью потенциала [8-10]. В случае сингулярного потенциала ситуация качественно меняется. Примеры показывают, что в этом случае последовательность $\left\{\gamma_{q}(n)\right\}_{n \in \mathbb{N}}$ может быть и неограничена. В связи с этим возникает вопрос о нахождении условий, при которых последовательность $\left\{\gamma_{q}(n)\right\}_{n \in \mathbb{N}}$ принадлежит классу $\mathbf{1}_{\infty}$ или $\mathbf{c}_{0}$. Его исследованию и посвящена данная работа.

Обозначим через $s(q)$ порядок гладкости произвольного распределения $q(\cdot)$ в гильбертовой шкале соболевских пространств на единичной окружности $\mathbb{T}$ :

$$
s(q):=\sup \left\{s \in \mathbb{R} \mid q \in H^{s}(\mathbb{T})\right\} \geqslant-1 .
$$

Как известно, периодическое распределение $q(\cdot)$ вида (2) принадлежит пространству Соболева $H^{s}(\mathbb{T})$ тогда и только тогда, когда

$$
\left\|\left.q\left|H^{s}(\mathbb{T}) \|^{2}=\sum_{k \in \mathbb{Z}}(1+2|k|)^{2 s}\right| \hat{q}(k)\right|^{2}<\infty .\right.
$$

Теорема 1. Пусть у оператора $S(q)$ порядок сингулярности потенциала $s(q) \in[-1,-1 / 2)$. Тогда последовательность длин спектральных лакун $\left\{\gamma_{q}(n)\right\}_{n \in \mathbb{N}}$ неограничена.

Доказательство теоремы использует известные асимптотические формулы для длин спектральных лакун. Пусть $q \in H^{s}(\mathbb{T}), s \in(-1,0]$, тогда согласно [12, теорема 1] мы имеем:

$$
\gamma_{q}(n)=2|\hat{q}(n)|+h^{1+2 s-\delta}(n) \quad \forall \delta>0 .
$$

Тут весовые пространства последовательностей $h^{s}(\mathbb{N}) \equiv h^{s}(\mathbb{N} ; \mathbb{C})$ определяются следующим образом:

$$
h^{s}(\mathbb{N}):=\left\{\left.\{a(k)\}_{k \in \mathbb{N}}\left|\sum_{k \in \mathbb{N}}(1+|k|)^{2 s}\right| a(k)\right|^{2}<\infty\right\},
$$


а через $h^{s}(n)$ обозначено $n$-й элемент последовательности, принадлежащей $h^{s}(\mathbb{N})$. Очевидно, что

$$
\{a(k)\}_{k \in \mathbb{N}} \in h^{s}(\mathbb{N}) \Rightarrow a(k)=o\left(k^{-s}\right), \quad k \rightarrow \infty .
$$

Нам также будет необходимо соотношение между гладкостью потенциала и скоростью изменения длин спектральных лакун [11, теорема 29] (см. также [12, 13]):

$$
q \in H^{s}(\mathbb{T}) \Leftrightarrow\left\{\gamma_{q}(n)\right\}_{n \in \mathbb{N}} \in h^{s}(\mathbb{N}), \quad s \in[-1, \infty)
$$

Доказательство проведем от противного. Предположим, что существует такой потенциал $q \in H^{-1}(\mathbb{T})$ с $s(q) \in(-1,-1 / 2)$, для которого соответствующая последовательность длин спектральных лакун $\left\{\gamma_{q}(n)\right\}_{n \in \mathbb{N}}$ является ограниченной, т. е., $\left\{\gamma_{q}(n)\right\}_{n \in \mathbb{N}} \in 1_{\infty}(\mathbb{N})$. Тогда очевидно, что $\left\{\gamma_{q}(n)\right\}_{n \in \mathbb{N}} \in h^{-1 / 2-\delta}(\mathbb{N}) \forall \delta>0$.

Тогда в силу (4) мы имеем $q \in H^{-1 / 2-\delta}(\mathbb{T}) \forall \delta>0$, т. е., $s(q) \geqslant-1 / 2$. Полученное противоречие доказывает ошибочность сделанного предположения.

Теорему 1 дополняет

Теорема 2. Пусть у оператора $S(q)$ порядок сингулярности потенщиала $s(q) \in(-1 / 2,0]$. тогда последовательность

$$
\gamma_{q}(n)-2|\hat{q}(n)| \in \mathbf{c}_{0} .
$$

В частности,

a) $\gamma_{q}(n) \in 1_{\infty}$ тогда и только тогда, когда потенциал $q(\cdot)$ является псевдомерой, m. $е$. $\{\hat{q}(k)\}_{k \in \mathbb{Z}} \in \mathbf{l}_{\infty}$;

б) условие $\gamma_{q}(n) \in \mathbf{c}_{0}$ равносильно тому, что $q(\cdot)$ является псевдофункцией, $m . е$ $\{\hat{q}(k)\}_{k \in \mathbb{Z}} \in \mathbf{c}_{0}$.

Доказательство. Пусть $s(q) \in(-1,2,0]$. Тогда $q \in H^{s(q)-\varepsilon}(\mathbb{T}) \forall \varepsilon>0$, и в силу формулы (3) мы имеем:

$$
\gamma_{q}(n)=2|\hat{q}(n)|+h^{1+2 s(q)-2 \varepsilon-\delta}(n) \quad \forall \varepsilon>0, \quad \forall \delta>0 .
$$

Поскольку $s(q)>-1 / 2$, то мы можем выбрать $\varepsilon>0$ и $\delta>0$ таким образом, чтобы выполнялось неравенство

$$
1+2 s(q)-2 \varepsilon-\delta \geqslant 0 .
$$

Тогда асимптотические оценки принимают вид

$$
\gamma_{q}(n)=2|\hat{q}(n)|+h^{0}(n)
$$

Но $h^{0}(n)=o(1), n \rightarrow \infty$. Поэтому из (6) следуют утверждения теоремы.

Следствие. Пусть $\left\{n_{k}\right\}_{k \in \mathbb{N}}-$ произвольная последовательность натуральньх чисел, $a$ $c \in[0,+\infty]$. Тогда если $s(q) \in(-1 / 2,0]$, то

$$
\gamma_{q}\left(n_{k}\right) \rightarrow 2 c \Leftrightarrow\left|\hat{q}\left(n_{k}\right)\right| \rightarrow c,
$$

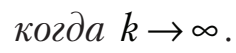


Теорема 2 позволяет строить примеры потенциалов, для которых последовательность длин спектральных лакун имеет заданные свойства. В частности, таких, что $s(q)=0$, но последовательность $\left\{\gamma_{q}(n)\right\}_{n \in \mathbb{N}} \notin \mathbf{l}_{\infty}$.

Пример. Пусть

$$
v(x)=\sum_{k \in \mathbb{Z}} \hat{v}(k) e^{i k 2 \pi x},
$$

где коэффищиенть ряда Фурье-Швариа определены следующим образом:

$$
\hat{v}(k):= \begin{cases}n, & \text { если }|k|=2^{n}, n \in \mathbb{N}, \\ 0, & \text { в противном случае. }\end{cases}
$$

Тогда

$$
\sum_{k \in \mathbb{Z}}|\hat{v}(k)|^{2}=2 \sum_{n \in \mathbb{N}} n^{2}=+\infty, \quad \text { m.e. }, \quad v \notin L^{2}(\mathbb{T}) .
$$

Однако для каждого $\delta>0$

$$
\sum_{k \in \mathbb{Z}}(1+2|k|)^{-2 \delta}|\hat{v}(k)|^{2}=2 \sum_{n \in \mathbb{N}}\left(1+2^{n+1}\right)^{-2 \delta} n^{2}<\infty, \quad \text { m.e., } \quad v \in H^{-\delta}(\mathbb{T}) .
$$

В силу теоремь 2 последовательность длин спектральных лакун неограничена, а в силу следствия из нее

$$
\begin{aligned}
& \lim _{M \ni n \rightarrow \infty} \gamma_{q}(n)=+\infty ; \\
& \lim _{M \ngtr n \rightarrow \infty} \gamma_{q}(n)=0,
\end{aligned}
$$

әде $M:=\left\{n \in \mathbb{N} \mid n=2^{k}, \quad k \in \mathbb{N}\right\}$

3. Вопрос о том, верна ли теорема 2 при $s(q)=-\frac{1}{2}$, остается пока открытым. Этот случай особо важен для физических приложений, так как вместе со случаем $s(q)>-\frac{1}{2}$ он охватывает все вещественные меры Радона на $\mathbb{R}$, которые являются обобщенными производными функций из класса $B V_{\mathrm{loc}}(\mathbb{R}) \subset L_{\mathrm{loc}}^{2}(\mathbb{R})$. Он требует отдельного исследования. Вместе с тем для мер нами установлена

Теорема 3. Последовательность длин спектральных лакун $\left\{\gamma_{q}(n)\right\}_{n \in \mathbb{N}}$ оператора Хилла-Шредингера $S(q)$ с потенциалом $q(\cdot)$, который является вещественной 1-периодической мерой Радона, ограничена

Доказательство теоремы 3 использует результат [14, теорема 12.8.1] и [15, теорема 1]. Оно будет приведено в другой публикации.

\section{ЦИТИРОВАННАЯ ЛИТЕРАТУРА}

1. Савчук А.М., Шкаликов А.А. Операторы Штурма Лиувилля с потенциалами-распределениями. Тр. Моск. мат. об-ва. 2003. 64. С. 159-212. 
2. Hryniv R.O., Mykytyuk Ya.V. 1-D Schrödinger operators with periodic singular potentials. Methods Funct. Anal. Topology. 2001. 7, № 4. P. 31-42.

3. Korotyaev E. Characterization of the spectrum of Schrödinger operators with periodic distributions. Int. Math. Res. Not. 2003. 37. P. 2019-2031. doi: https://doi.org/10.1155/S1073792803209107

4. Mikhailets V., Molyboga V. One-dimensional Schrödinger operators with singular periodic potentials. Methods Funct. Anal. Topology. 2008. 14, № 2. P. 184-200.

5. Djakov P., Mityagin B. Fourier method for one-dimensional Schrödinger operators with singular periodic potentials. Topics in Operator Theory. Operator Theory: Advances and Applications, Vol. 203. Basel: Birkhäuser, 2010. P. 195-236. doi: https://doi.org/10.1007/978-3-0346-0161-0 Ł9

6. Mikhailets V., Molyboga V. Singularly perturbed periodic and semiperiodic differential operators. Ukr. Math. J. 2007. 59, № 6. P. 858-873. doi: https://doi.org/10.1007/s11253-007-0055-7

7. Mikhailets V., Molyboga V. On the spectrum of singular perturbations of operators on the circle. Math. Notes. 2012. 91, № 3-4. P. 588-591. doi: https://doi.org/10.1134/S0001434612030352

8. Марченко В.А., Островский И.В. Характеристика спектра оператора Хилла. Матем. сб. 1975. 97, № 4. C. 540-606.

9. Djakov P., Mityagin B. Instability zones of periodic 1-dimensional Schrödinger and Dirac operators. Russ. Math. Surv. 2006. 61, № 4. P. 663-766. doi: https://doi.org/10.1070/RM2006v061n04ABEH004343

10. Mikhailets V., Molyboga V. Smoothness of Hill's potential and lengths of spectral gaps. Spectral Theory, Mathematical System Theory, Evolution Equations, Differential and Difference Equations. Operator Theory: Advances and Applications, Vol. 221. Basel: Birkhäuser, 2012. P. 469-479. doi: https://doi.org/10.1007/9783-0348-0297-0_27

11. Djakov P., Mityagin B. Spectral gaps of Schrödinger operators with periodic singular potentials. Dynam. Part. Differ. Eq. 2009. 6, № 2. P. 95-165. doi: https://doi.org/10.4310/DPDE.2009.v6.n2.a1

12. Mikhailets V., Molyboga V. Spectral gaps of the one-dimensional Schrödinger operators with singular periodic potentials. Methods Funct. Anal. Topology. 2009. 15, № 1. P. 31-40.

13. Mikhailets V., Molyboga V. Hill's potentials in Hörmander spaces and their spectral gaps. Methods Funct. Anal. Topology. 2011. 17, № 3. P. 235-243.

14. Atkinson F.V. Discrete and continuous boundary problems. Mathematics in Science and Engineering. Vol. 8. New York, London: Academic Press, 1964. xiv+570 pp.

15. Молибога В.Н. Характеризация спектральных лакун в спектре оператора Хилла с потенциаломраспределением. Зб. праць Інституту математики НАН України. 2013. 10, № 2. С. 248-259.

Поступило в редакцию 12.07.2018

\section{REFERENCES}

1. Savchuk, A. M. \& Shkalikov, A. A. (2003). Sturm-Liouville operators with distribution potentials. Tr. Mosk. mat. ob-va., 64, pp. 159-212 (in Russian).

2. Hryniv, R. O. \& Mykytyuk, Ya. V. (2001). 1-D Schrödinger operators with periodic singular potentials. Methods Funct. Anal. Topology, 7, No. 4, pp. 31-42.

3. Korotyaev, E. L. (2003). Characterization of the spectrum of Schrödinger operators with periodic distributions. Int. Math. Res. Not., 37, pp. 2019-20131. doi: https://doi.org/10.1155/S1073792803209107

4. Mikhailets, V. \& Molyboga, V. (2008). One-dimensional Schrödinger operators with singular periodic potentials. Methods Funct. Anal. Topology, 14, No. 2, pp. 184-200.

5. Djakov, P. \& Mityagin, B. (2010). Fourier method for one-dimensional Schrödinger operators with singular periodic potentials. In Topics in Operator Theory. Operator Theory: Advances and Applications (Vol. 203) (pp. 195-236). Basel: Birkhäuser. doi: https://doi.org/10.1007/978-3-0346-0161-0_9

6. Mikhailets, V. A. \& Molyboga, V. M. (2007). Singularly perturbed periodic and semiperiodic differential operators. Ukr. Math. J., 59, No. 6, pp. 858-873. doi: https://doi.org/10.1007/s11253-007-0055-7

7. Mikhailets, V. A. \& Molyboga, V. (2012). On the spectrum of singular perturbations of operators on the circle. Math. Notes, 91, No. 3-4, pp. 588-591. doi: https://doi.org/10.1134/S0001434612030352

8. Marčenko, V. A \& Ostrovs'kiǐ, I. V. (1975). A characterization of the spectrum of the Hill operator. Mat. USSR-Sb., 26, No. 4, pp. 493-554. 
9. Djakov, P. \& Mityagin, B. (2006). Instability zones of periodic 1-dimensional Schrödinger and Dirac operators. Russ. Math. Surv., 64, No. 4, pp. 663-766. doi: https://doi.org/10.1070/RM2006v061n04ABEH004343

10. Mikhailets, V. \& Molyboga, V. (2012). Smoothness of Hill's potential and lengths of spectral gaps. Spectral Theory, Mathematical System Theory, Evolution Equations, Differential and Difference Equations. Operator Theory: Advances and Applications (Vol.221) (pp. 469-479). Basel: Birkhäuser. doi: https://doi.org/10.1007/ 978-3-0348-0297-0_27

11. Djakov, P. \& Mityagin, B. (2009). Spectral gaps of Schrödinger operators with periodic singular potentials. Dynam. Part. Differ. Eq., 6, No. 2, pp. 95-165. doi: https://doi.org/10.4310/DPDE.2009.v6.n2.a1

12. Mikhailets, V. \& Molyboga, V. (2009). Spectral gaps of the one-dimensional Schrödinger operators with singular periodic potentials. Methods Funct. Anal. Topology, 15, No. 1, pp. 31-40.

13. Mikhailets, V. \& Molyboga, V. (2011). Hill's potentials in Hörmander spaces and their spectral gaps. Methods Funct. Anal. Topology, 17, No. 3, pp. 235-243.

14. Atkinson, F. V.(1964). Discrete and continuous boundary problems. Mathematics in Science and Engineering, Vol. 8. New York, London: Academic Press.

15. Molyboga, V. (2013). Characterization of spectral gapsin the spectrum of Hill's operator with distributional potential. Zb. Prats Instytutu matematyky NAN Ukrajiny, 10, No. 2, pp. 248-259 (in Russian).

Received 12.07.2018

\section{В.А. Михайлеиь, В.М. Молибога}

Інститут математики НАН України, Київ

E-mail: mikhailets@imath.kiev.ua, molyboga@imath.kiev.ua

ПРО ЛАКУНИ В СПЕКТРІ ОПЕРАТОРА

ХІЛЛА-ШРЕДІНГЕРА З СИНГУЛЯРНИМ ПОТЕНЦІАЛОМ

Досліджується неперервний спектр оператора Хілла-Шредінгера в гільбертовому просторі $L^{2}(\mathbb{R})$. Вважається, що потенціал оператора належить до класу Соболєва $H_{\mathrm{loc}}^{-1}(\mathbb{R})$. Знайдено умови, за яких послідовність довжин спектральних лакун: а) обмежена; б) прямує до нуля. Окремо досліджено випадок, коли потенціал є дійсною мірою Радона на $\mathbb{R}$.

Ключові слова: оператор Хілла, неперервний спектр, спектральна лакуна, сильно сингулярний потенціал.

\section{V.A. Mikhailets, V.M. Molyboga}

Institute of Mathematics of the NAS of Ukraine, Kiev

E-mail: mikhailets@imath.kiev.ua,molyboga@imath.kiev.ua

\section{ON SPECTRAL GAPS OF THE HILL-SCHRÖDINGER OPERATOR WITH SINGULAR POTENTIAL}

We study the continuous spectrum of the Hill-Schrödinger operator in a Hilbert space $L^{2}(\mathbb{R})$. The operator potential belongs to a Sobolev space $H_{\text {loc }}^{-1}(\mathbb{R})$. The conditions are found for the sequence of lengths of spectral gaps to: a) be bounded; b) converge to zero. The case where the potential is a real Radon measure on $\mathbb{R}$ is studied separately.

Keywords: Hill's operator, continuous spectrum, spectral gap, strongly singular potential. 